\title{
The demise of superfluid density in overdoped La2-xSrxCuO4 films grown by molecular beam epitaxy
}

\author{
I. Božović, X. He1, J. Wu and A. T. Bollinger \\ Submitted to Journal of Superconductivity and Novel Magnetism \\ September 2016 \\ Condensed Matter Physics and Material Science Department \\ Brookhaven National Laboratory
}

\section{U.S. Department of Energy \\ USDOE Office of Science (SC), Basic Energy Sciences (BES) (SC-22)}

Notice: This manuscript has been authored by employees of Brookhaven Science Associates, LLC under Contract No. DE-SC0012704 with the U.S. Department of Energy. The publisher by accepting the manuscript for publication acknowledges that the United States Government retains a non-exclusive, paid-up, irrevocable, world-wide license to publish or reproduce the published form of this manuscript, or allow others to do so, for United States Government purposes. 


\section{DISCLAIMER}

This report was prepared as an account of work sponsored by an agency of the United States Government. Neither the United States Government nor any agency thereof, nor any of their employees, nor any of their contractors, subcontractors, or their employees, makes any warranty, express or implied, or assumes any legal liability or responsibility for the accuracy, completeness, or any third party's use or the results of such use of any information, apparatus, product, or process disclosed, or represents that its use would not infringe privately owned rights. Reference herein to any specific commercial product, process, or service by trade name, trademark, manufacturer, or otherwise, does not necessarily constitute or imply its endorsement, recommendation, or favoring by the United States Government or any agency thereof or its contractors or subcontractors. The views and opinions of authors expressed herein do not necessarily state or reflect those of the United States Government or any agency thereof. 


\title{
The demise of superfluid density in overdoped $\mathrm{La}_{2-x} \mathrm{Sr}_{\underline{x}} \mathrm{CuO}_{4}$ films grown by molecular beam epitaxy
}

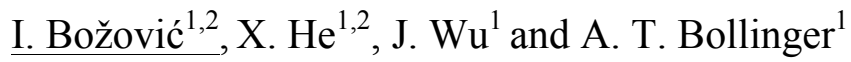

${ }^{1}$ Brookhaven National Laboratory, Upton, New York 11973-5000, USA

${ }^{2}$ Yale University, Department of Applied Physics, New Haven CT 06511, USA

\section{Corresponding Author:}

Ivan Božović

Phone: $\quad$ (631) 344-4973

Fax: $\quad$ (631) 344-4071

E-mail: $\quad$ bozovic@bnl.gov

\section{Keywords}

High temperature superconductivity, cuprate, thin films, molecular beam epitaxy, superfluid density

\begin{abstract}
We have synthesized $\mathrm{La}_{2-x} \mathrm{Sr}_{x} \mathrm{CuO}_{4}$ thin films using atomic-layer-by-layer molecular beam epitaxy (ALL-MBE). The films are high-quality — singe crystal, atomically smooth, and very homogeneous. The critical temperature $\left(T_{c}\right)$ shows very little $(<1 \mathrm{~K})$ variation within a film of $10 \times 10 \mathrm{~mm}^{2}$ area. The large statistics (over 2,000 films) is crucial to discern intrinsic properties. In these films, we have measured the absolute value of the magnetic penetration depth $\lambda$ with the accuracy better than $1 \%$, and mapped densely the entire overdoped side of the $\mathrm{La}_{2-x} \mathrm{Sr}_{x} \mathrm{CuO}_{4}$ phase diagram. A new scaling law is established accurately for the dependence of $T_{c}$ on the superfluid density. The scaling we observe is incompatible with the standard Bardeen-CooperSchrieffer picture, and points to local pairing.
\end{abstract}




\section{Introduction}

Underdoped cuprate superconductors have been studied in great detail. ${ }^{1-3}$. The overdoped side has received much less attention. It seems to be almost generally believed that with overdoping the physics evolves towards the conventional one, where the normal state can be well described by the Landau Fermi liquid theory and the superconducting state by the Bardeen-CooperSchrieffer (BCS) theory. In variance with this simple picture, several early studies ${ }^{4-9}$ on a few overdoped samples showed that the measured superfluid density was much smaller than what one would expect in a clean BCS superconductor. However, this discrepancy was attributed to extrinsic factors such as phase separation ${ }^{4}$ or pair-breaking $^{9}$, and largely brushed away.

\section{Experimental methods}

We have studied this question in great detail by measuring the dependence of the magnetic penetration depth $\lambda$ on temperature and doping in overdoped $\mathrm{La}_{2-x} \mathrm{Sr}_{x} \mathrm{CuO}_{4}$ (LSCO) films. For film synthesis, we used a unique atomic-layer-by-layer molecular beam epitaxy (ALL-MBE) system at Brookhaven National Laboratory that provides single-crystal thin films free of usual defects such as secondary-phase precipitates, grain boundaries, pinholes, etc ${ }^{10-12}$. The total number of LSCO films we have studied, in ten years, is well over 2,000, and this large statistics is critically important since it allows us to clearly identify the trends and systematics, and thus to differentiate between intrinsic behavior and the effects of various extrinsic factors. The sample homogeneity can be judged by the sharpness of the superconducting transition indicated by the onset of the Meissner effect. In our best films, $T_{c}$ is homogeneous on the scale of $\sim 0.1 \mathrm{~K}$, over the film area of $10 \times 10 \mathrm{~mm}^{2}$.

We have measured $\lambda(T, x)$ using the two-coil mutual inductance technique ${ }^{13-19}$. To improve the accuracy, we have made a number of technical improvements in the apparatus and the procedure $^{20}$, ensuring great reproducibility and precision (better than $0.3 \%$ ) in the raw data. To extract from these the absolute values of $\lambda$, a critical requirement is that the thickness of the superconducting layer must be accurately known. To meet this goal, we have used ALL-MBE to engineer the samples at the atomic-layer level (including $\delta$-doping with $\mathrm{Zn}$ ), synthesizing multilayer structures in which the active (superconducting) layer thickness is sharply defined. Altogether, we measure the absolute value of $\lambda$ with the accuracy of $\pm 1 \%$. 


\section{Experimental results}

In Fig. 1, we show the doping dependence of $\lambda(T)$ covering densely the entire overdoped side of the LSCO phase diagram. These data are extracted directly from the measured raw inductance data; the only sample-dependent input parameter is the film thickness, which we know accurately by virtue of our atomic-layer-by-layer ('digital') synthesis technique.

For few most heavily overdoped films, in Fig. 2 we show the corresponding 2D superfluid density $N_{s}$, expressed as the number of holes per one $\mathrm{CuO}_{2}$ unit. It is determined as $N_{s} \equiv A / \lambda^{2}$, where $A$ $\equiv m^{*} a_{0}^{2} d / \mu_{0} e^{2}, m^{*}$ is the effective mass, $a_{0}=0.38 \mathrm{~nm}$ is the in-plane lattice constant, $d=0.662$ $\mathrm{nm}$ is the distance between two neighbor $\mathrm{CuO}_{2}$ planes, $\mu_{0}=4 \pi \times 10^{-7} \mathrm{~N} / \mathrm{A}^{2}$ is the vacuum permeability, and $e$ is the electron charge. In this plot, we have assumed for simplicity that $m^{*}=2 m_{e}$, independent on doping; this is the largest value compatible with our data at optimal doping, and it gives $A=5.14 \times 10^{-15} \mathrm{~m}^{2}$. Note that this choice is made just to facilitate communication and comparison with the sample doping level, which is usually also expressed as a number of holes per $\mathrm{Cu}$. We can equally well formulate all of our conclusions ${ }^{20}$ without any assumptions, in terms of the superfluid phase stiffness, defined as $\rho_{s} \equiv\left(4.15 \times 10^{-12} \mathrm{~K}-\mathrm{m}^{2}\right) / \lambda^{2}$.

As seen from Fig. 2, the $N_{s}(T)$ curves are essentially linear, apart from some small deviations from linearity at very low $T$ and/or also very close to $T_{c}$. This is indeed expected theoretically ${ }^{21}$ and observed experimentally ${ }^{22,23}$ in very clean d-wave superconductors. However, the absolute values of $N_{s 0} \equiv N_{s}(T \rightarrow 0)$ are unexpectedly extremely small, as low as $1 \%$ of the total mobile carrier density (if we assume that the later is equal to $p$, and even less if we equate it with $1+p$, as frequently postulated). Since $N_{s 0}$ cannot be measured directly, but only extrapolated from the values measured down to the experimental limit, there is some uncertainty due to this extrapolation. However, note that our measurements extend down to $T=300 \mathrm{mK}$, so this error is generally negligible. This is illustrated in the inset to Figure 2, for two samples, one very highly overdoped with a low $T_{c} \approx 5 \mathrm{~K}$, and the other much closer to the optimal doping, with $T_{c} \approx 36 \mathrm{~K}$. (The corresponding doping levels, $p=0.255$ and $p=0.195$, are only nominal, and used just for communication $-p$ is not a directly measured quantity ${ }^{20}$.) Both are fitted to $N_{s}(T)=N_{s 0}-A T-B T^{2}$, and the fits are essentially perfect $(\mathrm{R}=0.99981$ and $\mathrm{R}=0.99999$, respectively). More to the point, the difference between the extrapolated $N_{s 0}$ and the closest measured value $N_{s}(T=300 \mathrm{mK})$ is 
very small to begin with at any doping $\left(\Delta N_{s}=0.0002\right.$ for $\mathrm{p}=0.255$ and $\Delta N_{s}=0.0005$ for $\mathrm{p}=$ 0.195 film, respectively). The differences between the values extrapolated using different functional forms (e.g., strictly linear or strictly quadratic) are even smaller, on the order of $1 \%$ in the worst case (the lowest $T_{c}$ ) and much less elsewhere.

Fig. 3 shows the dependence of $T_{c}$ on $N_{s 0}$. For the most part, the $T_{c}\left(N_{s 0}\right)$ scaling in Fig. 3 is linear but with an offset, $T_{c}=T_{0}+\alpha N_{s 0}$, with $T_{0}=(7.0 \pm 0.1) \mathrm{K}$ and $\alpha=(2.5 \pm 0.1) \times 10^{2} \mathrm{~K}$. This scaling breaks down very close to the origin, i.e., for $N_{s 0}<0.02$ or so; there, the curve can be fitted well to $T_{c}=\gamma \sqrt{ } N_{s 0}$ with $\gamma=(1.1 \pm 0.1) \times 10^{2} \mathrm{~K}$. Note that in this figure the error bars are smaller than the marker size.

\section{Conclusions}

This accurate scaling law imposes a very tight constraint on the theory. We have gathered and presented $^{20}$ ample evidence that it cannot be explained by assuming impurities and disorder within the standard dirty-d-wave-BCS picture. The precise form of this $T_{c}\left(N_{s 0}\right)$ scaling is novel and surprising, and it challenges the prevalent creeds ${ }^{24,25}$. An important inference is that $T_{c}$ appears to be essentially controlled by the superfluid density, i.e., by the kinematics. This is more consistent with local pairing rather than with the BCS picture, in which $T_{c}$ is determined by dynamics of electrons and phonons, and their interaction. Note, however, that for any single-species system that is translation- and time reversal-invariant, the superfluid density must be equal to the total

particle density, as shown by A. Leggett ${ }^{26,27}$. The fact that this law is so dramatically violated in samples that are evidently very homogeneous and clean poses a serious problem to the theory. We may need to rethink some of our basic premises.

\section{References}

1. Lee, P. A., Nagaosa, N., Wen, X. G.: Doping a Mott insulator: Physics of high-temperature superconductivity. Rev. Mod. Phys. 78, 17-85 (2006).

2. Zaanen, J., Chakravarty, S., Senthil, T., Anderson, P. W., Lee, P., Schmalian, J., Imada, M., Pines, D., Randeria, M., Varma, C., Vojta, M., Rice, M.: Towards a complete theory of high $\mathrm{T}_{\mathrm{c}}$. Nature Phys. 2, 138143 (2006).

3. Keimer, B., Kivelson, S. A., Norman, M. R., Uchida, S., Zaanen, J.: From quantum matter to hightemperature superconductivity in copper oxides. Nature 518, 179-186 (2015).

4. Uemura, Y. J. et al.: Universal correlations between $\mathrm{T}_{\mathrm{c}}$ and $\mathrm{n}_{\mathrm{s}} / \mathrm{m}^{*}$ (carrier density over effective mass) in high- $\mathrm{T}_{\mathrm{c}}$ cuprate superconductors. Phys. Rev. Lett. 62, 2317-2320 (1989). 
5. Uemura, Y. J. et al.: Magnetic-field penetration depth in $\mathrm{TI}_{2} \mathrm{Ba}_{2} \mathrm{CuO}_{6+\delta}$ in the overdoped regime. Nature 364, 605-607 (1993).

6. Niedermayer, C. et al:: Muon spin rotation study of the correlation between $\mathrm{T}_{\mathrm{c}}$ and $\mathrm{n}_{\mathrm{s}} / \mathrm{m}^{*}$ in overdoped $\mathrm{Tl}_{2} \mathrm{Ba}_{2} \mathrm{CuO}_{6+\delta}$. Phys. Rev. Lett. 71, 1764-1767 (1993).

7. Bernhard, C. et al.: Magnetic penetration depth and condensate density of cuprate high- $\mathrm{T}_{\mathrm{c}}$ superconductors determined by muon-spin-rotation experiments. Phys. Rev. B 52, 10488-10498 (1995).

8. Panagopoulos, C. et al.: Superfluid response in monolayer high- $\mathrm{T}_{\mathrm{c}}$ cuprates. Phys. Rev. B 67, 220502 (2003).

9. Tallon, J. L., Loram, J. W., Cooper, J. R., Panagopoulos, C., Bernhard, C.: Superfluid density in cuprate high- $\mathrm{T}_{\mathrm{c}}$ superconductors: A new paradigm. Phys. Rev. B 68, 180501 (2003).

10. Bozovic, I.: Atomic-layer engineering of superconducting oxides: Yesterday, today, tomorrow. IEEE Trans. Appl. Supercond. 11, 2686-2695 (2001).

11. Gozar, A. et al.: Interface superconductivity between a metal and a Mott insulator. Nature 455, 782-785 (2008).

12. Logvenov, G., Gozar, A., Bozovic, I.: High-temperature superconductivity in a single copper-oxygen plane. Science 326, 699-702 (2009).

13. Hebard, A. F., Fiory, A. T.: Evidence for the Kosterlitz-Thouless transition in thin superconducting aluminum films. Phys. Rev. Lett. 44, 291-294 (1980).

14. Claassen, J. H., Reeves, M. E., Soulen, R. J. Jr.: A contactless method for measurement of the critical current density and critical temperature of superconducting rings. Rev. Sci. Instrum. 62, 996-1004 (1991).

15. Clem, J. R., Coffey, M. W.: Vortex dynamics in a type-II superconducting film and complex linear-response functions. Phys. Rev. B 46, 14662-14674 (1992).

16. Turneaure, S. J., Ulm, E. R., Lemberger, T. R.: Numerical modeling of a two-coil apparatus for measuring the magnetic penetration depth in superconducting films and arrays. J. Appl. Phys. 79, 4221-4227 (1996).

17. Locquet, J. P. et al:: Variation of the in-plane penetration depth $\lambda_{\mathrm{ab}}$ as a function of doping in $\mathrm{La}_{2-\mathrm{x}} \mathrm{Sr}_{\mathrm{x}} \mathrm{CuO}_{4+\delta}$ thin films on $\mathrm{SrTiO}_{3}$ : implications for the overdoped state. Phys. Rev. B 54, 7481-7488 (1996).

18. Turneaure, S. J., Pesetski, A. A., Lemberger, T. R.: Numerical modeling and experimental considerations for a two-coil apparatus to measure the complex conductivity of superconducting films. J. Appl. Phys. 83, 43344343 (1998).

19. Lemberger, T. R., et al.: Superconductor-to-metal quantum phase transition in overdoped $\mathrm{La}_{2-\mathrm{x}} \mathrm{Sr}_{\mathrm{x}} \mathrm{CuO}_{4}$. Phys. Rev. B 83, 140507 (2011).

20. Božović, I., He, X., Wu, J., Bollinger, A. T.: Dependence of critical temperature in overdoped copper oxides on superfluid density. Nature 536, 309-311 (2016).

21. Hirschfeld, P. J., Goldenfeld, N.: Effect of strong scattering on the low-temperature penetration depth of a dwave superconductor. Phys. Rev. B 48, 4219-4222 (1993).

22. Hosseini, A. et al.: Microwave spectroscopy of thermally excited quasi-particles in $\mathrm{YBa}_{2} \mathrm{Cu}_{3} \mathrm{O}_{6.99}$. Phys. Rev. B 60, 1349-1359 (1999).

23. Broun, D. M. et al.: Superfluid density in a highly underdoped $\mathrm{YBa}_{2} \mathrm{Cu}_{3} \mathrm{O}_{6+\mathrm{y}}$ superconductor. Phys. Rev. Lett. 99, 237003 (2007).

24. Zaanen, J.: Superconducting electrons go missing. Nature 536, 282-283 (2016).

25. Kivelson, S. A.: On the character of the superconductor to metal transition in overdoped cuprates. Journal Club for Condensed Matter Physics, 18 August 2016.

26. Leggett, A.: Quantum Liquids (Oxford Univ. Press, 2006), chapters 3.3.and 3.4. 
27. Leggett, A.: On the superfluid fraction of an arbitrary many-body system at T=0. J. Stat. Phys. 93, 927 (1998).

\section{Acknowledgements:}

This research was done at Brookhaven National Laboratory and was supported by the U.S. Department of Energy, Basic Energy Sciences, Materials Sciences and Engineering Division. X.H. is supported by the Gordon and Betty Moore Foundation's EPiQS Initiative through Grant GBMF4410. 


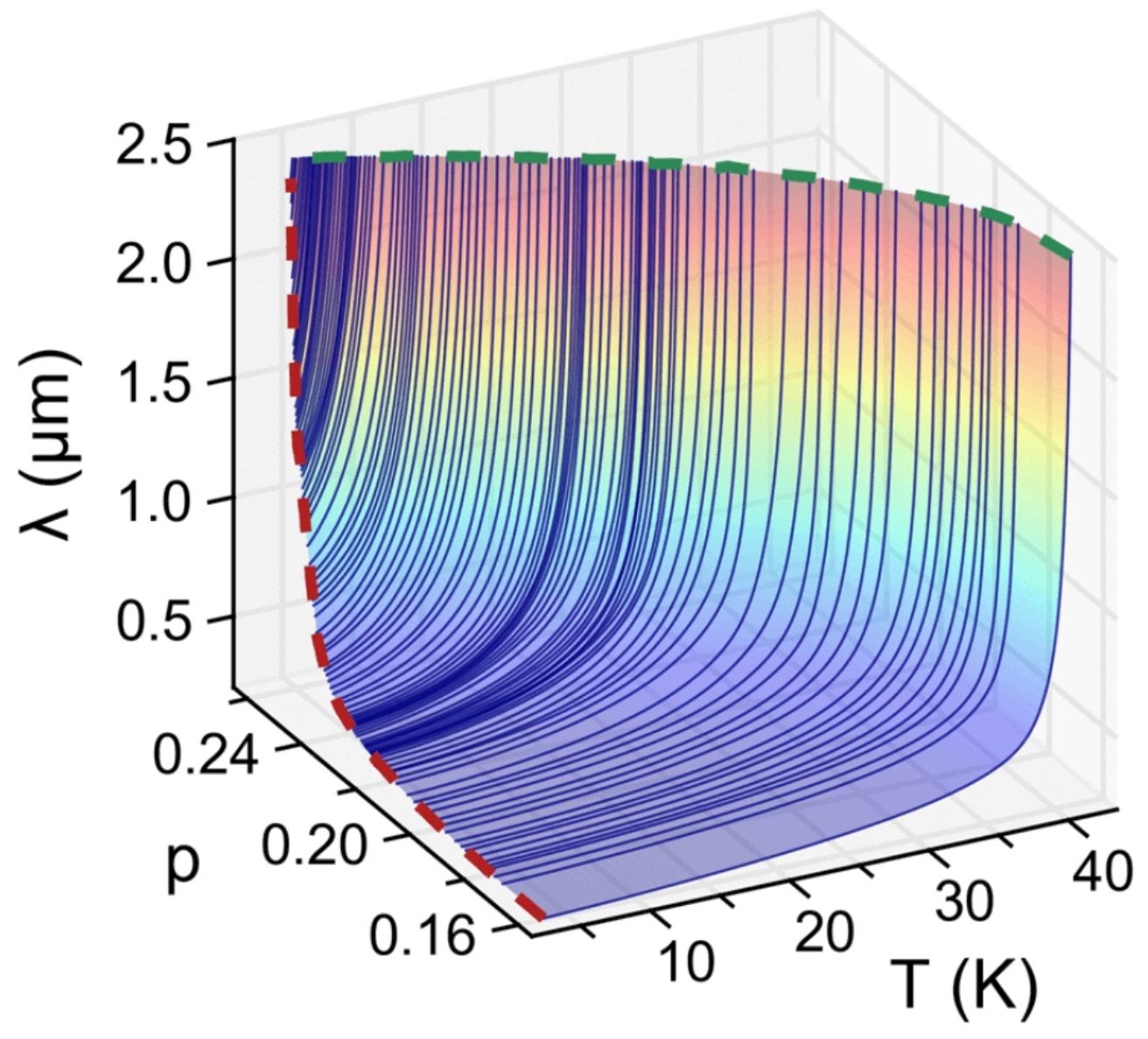

Fig. 1. The dependence of the magnetic penetration depth $(\lambda)$ on temperature $(T)$ and doping level $(p)$ in overdoped LSCO films synthesized by ALL-MBE. Note that $p$ is not directly measured but rather (as it is customary in this field) inferred from the measured value of $T_{c}$ assuming a phenomenological parabolic $T_{c}(p)$ dependence $^{20}$. 


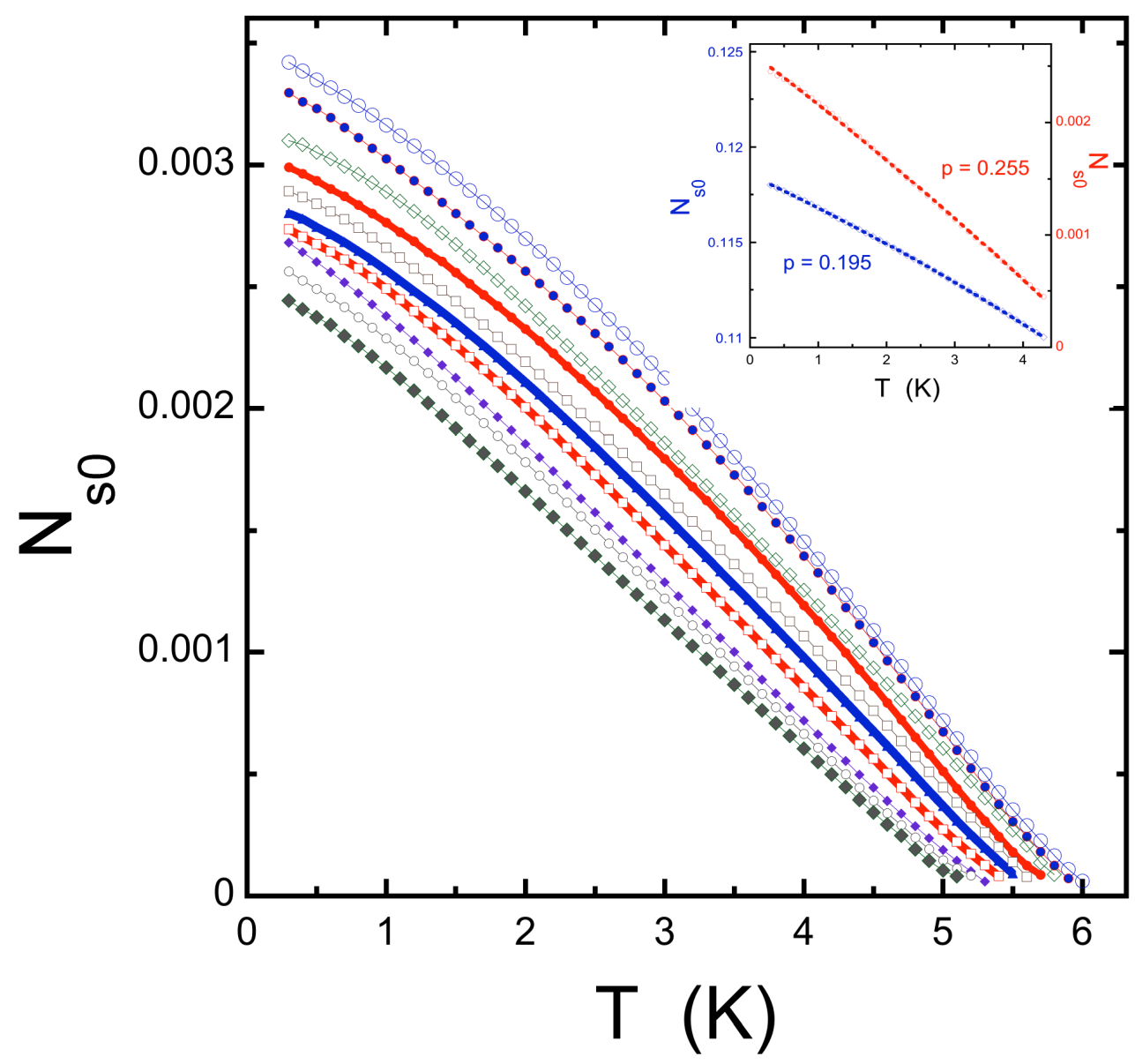

Fig. 2. The temperature and doping dependence of the in-plane superfluid density $N_{s} \equiv A / \lambda^{2}$, where $A=5.4 \times 10^{-15} \mathrm{~m}^{2}$, for ten heavily overdoped LSCO films grown by ALL-MBE. Note that it in the most overdoped film $N_{s 0}$ has dropped to $1 \%$ of the total mobile carrier (hole) density. The inset shows the fits to $N_{s}(T)$ $=N_{s 0}-A T-B T^{2}$, for two representative but widely different doping levels. 


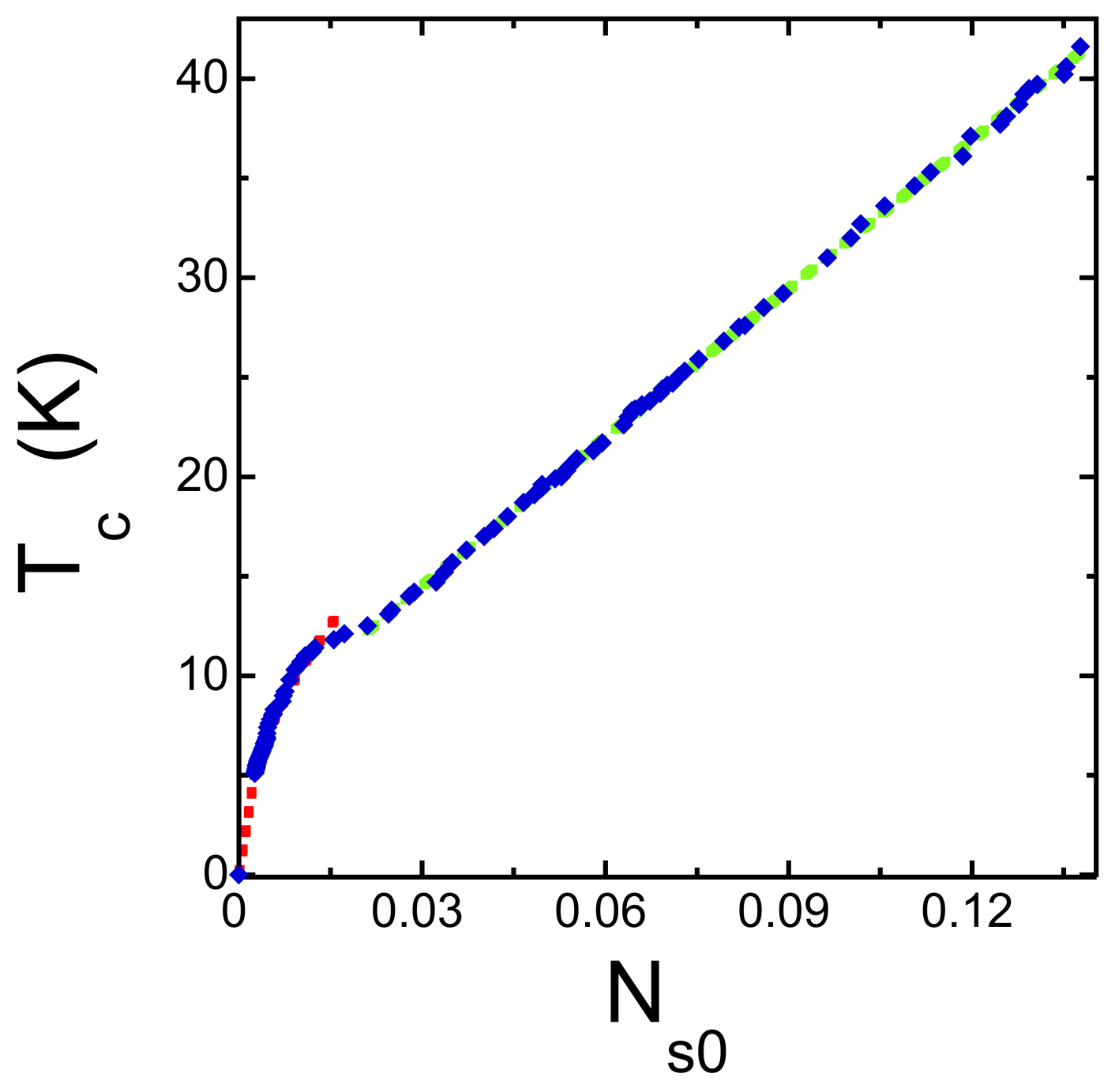

Fig. 3. Solid blue diamonds: the experimental data showing the dependence of $T_{c}$ on zero-temperature superfluid density $N_{s 0} \equiv N_{s}(T \rightarrow 0)$. The green dashed line: fit to $T_{c}=T_{0}+\alpha N_{s 0}$, where $T_{0}=(7 \pm 0.1) \mathrm{K}$, and $\alpha=(2.5 \pm 0.1) \times 10^{2} \mathrm{~K}$, for $N_{s 0}>$ 0.02. The red dashed line: fit to $T_{c}=\gamma \sqrt{ } N_{s 0}$, with $\gamma=(1.1 \pm 0.1) \times 10^{2} \mathrm{~K}$, for $N_{s 0}<$ 0.02 . 